\title{
How to Construct Pseudo-random Permutations from Pseudo-random Functions
}

\author{
Michael Luby \\ Charles Rackoff \\ Department of Computer Science \\ University of Toronto \\ Toronto, Canada M5S 1A4
}

\begin{abstract}
Let $F^{n}$ be the set of all functions from $n$ bits to $n$ bits. Let $f^{n}$ specify or each key $k$ of a given length a function $f_{k}^{n} \in F^{n}$. We say $f^{n}$ is pseudorandom if the following two properties hold:

(1) Given a key $k$ and an input $\alpha$ of length $n$, the time to evaluate $f_{k}^{n}(\alpha)$ is polynomial in $n$.

(2) If a random key $k$ is chosen, $f_{k}^{n}$ "looks like" a random function chosen from $F^{n}$ to any algorithm which is allowed to evaluate $f_{k}^{n}$ at polynomial in $n$ input values.

Let $P^{2 n}$ be the set of permutations (1-1 onto functions) from $2 n$ bits to $2 n$ bits. Let $p^{2 n}$ specify for each key $k$ of a given length a permutation $p_{k}{ }^{2 n} \in P^{2 n}$. We present a simple method for describing $p^{2 n}$ in terms of $f^{n}$. The method has the property that if $f^{n}$ is pseudo-random then $p^{2 n}$ is also pseudo-random. The method was inspired by a study of the security of the Data Encryption Standard. This result, together with the result of Goldreich, Goldwasser and Micali [GGM], implies that if there is a pseudo-random number generator then there is a pseudo-random invertible permutation generator. We also prove that if two permutation generators which are "slightly secure" are cryptographically composed, the result is more secure than either one alone.
\end{abstract}

